6. Del Pozo L, Silva N, Valencia A, Soto J, Riveros JC, Sacsaquispe $\mathrm{R}$, et al. Estudio de un brote intrahospitalario por Salmonella typhimurium productora de beta-lactamasa de espectro extendido SHV-5. An Fac Med Lima 2006;67(4): 318-26.

Correspondencia: Edgar Gonzales Escalante,

Dirección: Av. Brasil 600, Lima 05, Perú.

Teléfono: (511) 330-0066 anexo 3201

Correoelectrónico:egones_5@hotmail.com

\section{DENGUE EN ICA: UNA OPORTUNIDAD PARA INTERVENCIONES EFECTIVAS}

\section{DENGUE IN ICA: AN OPPORTUNITY FOR EFFECTIVE INTERVENTIONS}

\section{Ubaldo Miranda-Soberón ${ }^{1, a}$, Tania Acevedo-Villar2,b, Alfredo León-Alejo ${ }^{2, b}$, Junior Torres-Román ${ }^{2, b}$}

Sr. Editor. Hasta mayo de 2015, la provincia de Ica era considerada escenario epidemiológico I para dengue, junto con otras regiones del sur del Perú. Sin embargo, debido a dos casos confirmados, que se ha demostrado que fueron importados, se realizó una investigación entomológica (2 de junio de 2015), confirmando la presencia de Aedes aegypti en diferentes distritos de la localidad. Algunos de ellos con un índice larvario alto (1), lo cual nos ubica en el escenario epidemiológico II.

En Perú, el dengue es producido por cuatro serotipos virales diferenciables. Considerando que en la mayoría de distritos de lca ya existe la presencia del vector, la probabilidad de la introducción de cualquiera de los serotipos virales es preocupante, ya que algunos están asociados con formas graves y mortales de la enfermedad, incluso las formas asintomáticas e inaparentes serían probables reservorios para el inicio de una epidemia en Ica (2). Se ha reportado casos de pacientes con infección asintomática que han desarrollado viremia significativa ${ }^{(3)}$.

Debido al rápido avance de la infestación aédica en el Perú, y considerando el gran tráfico de personas entre el

\footnotetext{
1 Dirección Regional de Salud de Ica. Ica, Perú.

2 Sociedad Científica de Estudiantes de Medicina de Ica (SOCEMI), Facultad de Medicina. Universidad Nacional San Luis Gonzaga. Ica, Perú.

a Médico pediatra; ${ }^{b}$ estudiante de Medicina

Recibido: 10-07-15 Aprobado: 23-07-15
}

Citar como: Miranda-Soberón U, Acevedo-Villar T, León-Alejo A, Torres-Román J. Dengue en Ica: una oportunidad para intervenciones efectivas [carta]. Rev Peru Med Exp Salud Publica. 2015;32(3):607-8. norte, Lima e Ica, además de la ruta de las poblaciones selváticas, Ayacucho e Ica, en el 2010 se realizó una investigación para identificar los conocimientos y prácticas sobre el dengue en el poblador iqueño. Se aplicó una encuesta a 681 personas de diferentes estratos socioeconómicos, de su lectura, se infiere que en algunas zonas ya se estaban dando las condiciones óptimas para la infestación aédica; lo cual solo era cuestión de tiempo (Tabla 1).

Tabla 1. Conocimientos, actitudes y prácticas sobre el dengue en tres estratos de pobladores de Ica, 2010

\begin{tabular}{|c|c|c|c|}
\hline & Residencial & $\begin{array}{l}\text { Casas cerca } \\
\text { a cementerio }\end{array}$ & $\begin{array}{c}\text { Asenta- } \\
\text { miento } \\
\text { humano }\end{array}$ \\
\hline & $\mathrm{N}=243(\%)$ & $\mathrm{N}=193(\%)$ & $\mathrm{N}=245(\%)$ \\
\hline $\begin{array}{l}\text { Fuente de Informa- } \\
\text { ción sobre dengue: } \\
\text { televisión }\end{array}$ & $64(26,3)$ & $81(41,9)$ & $74(30,2)$ \\
\hline $\begin{array}{l}\text { Conoce transmisión: } \\
\text { picadura de mosquito }\end{array}$ & $142(58,4)$ & $98(50,8)$ & $98(40,0)$ \\
\hline Usa repelentes & $97(39,9)$ & $61(31,6)$ & $57(23,3)$ \\
\hline $\begin{array}{l}\text { No identifica lugares } \\
\text { de desarrollo del } \\
\text { mosquito }\end{array}$ & $32(13,2)$ & $49(25,4)$ & $85(34,7)$ \\
\hline $\begin{array}{l}\text { Identifica como } \\
\text { lugares de desarrollo } \\
\text { del mosquito: baldes y } \\
\text { tanques }\end{array}$ & $39(16,1)$ & $26(13,5)$ & $36(14,7)$ \\
\hline $\begin{array}{l}\text { Identifica como } \\
\text { lugares de desarrollo } \\
\text { del mosquito: botellas, } \\
\text { floreros, macetas y } \\
\text { tiestos }\end{array}$ & $24(9,9)$ & $7(3,6)$ & $8(3,3)$ \\
\hline $\begin{array}{l}\text { Considera gravedad } \\
\text { del dengue }\end{array}$ & $113(46,5)$ & $90(46,6)$ & $89(36,3)$ \\
\hline $\begin{array}{l}\text { Asocia dengue con } \\
\text { fiebre }\end{array}$ & $72(29,6)$ & $41(21,2)$ & $29(11,8)$ \\
\hline $\begin{array}{l}\text { Asocia dengue con } \\
\text { fiebre y otros } \\
\text { síntomas }\end{array}$ & $48(19,7)$ & $46(23,8)$ & $68(27,8)$ \\
\hline $\begin{array}{l}\text { Recibe agua: red } \\
\text { pública }\end{array}$ & $239(98,4)$ & $191(98,9)$ & $166(67,8)$ \\
\hline $\begin{array}{l}\text { Usa envases limpios } \\
\text { con tapa }\end{array}$ & $178(73,2)$ & $179(92,7)$ & $215(87,8)$ \\
\hline Usa cisterna & $28(11,5)$ & $2(1,0)$ & $3(1,2)$ \\
\hline Uso cualquier envase & $4(1,6)$ & $2(1,0)$ & $8(3,3)$ \\
\hline $\begin{array}{l}\text { Limpieza de envases } \\
\text { :agua, lejía y escobilla }\end{array}$ & $176(72,4)$ & $123(63,7)$ & $159(64,9)$ \\
\hline $\begin{array}{l}\text { Vivienda : material } \\
\text { Noble }\end{array}$ & $239(98,3)$ & $123(63,7)$ & $91(37,1)$ \\
\hline
\end{tabular}

Este estudio determinó que la población iqueña estaba poco informada sobre las características, prevención y consecuencias del dengue. Muchos señalaron que se debía almacenar agua en envases con tapa, pero pocos mencionaron la frecuencia correcta de limpieza de los envases (entre 39,1 y 5,4 días), y muy pocos reconocían estos depósitos como criaderos. Esto evidenció la importancia de las capacitaciones sobre la limpieza de los contenedores de agua, sobre lo cual muy poco se ha avanzado. 
Existe presencia de Aedes aegypti en Ica y es necesario eliminarlo. No es competencia solo del sector salud. Se debe considerar como actores principales la participación comunitaria, de las autoridades, así como de las universidades y colegios, propiciando y reforzando la capacitación, comunicación y colaboración intersectorial ${ }^{(4)}$. La vigilancia respecto a la propagación del dengue debería ser conducida con una novedosa perspectiva orientada a la erradicación del vector y adecuado manejo de los casos que puedan presentarse (5). Se debe implementar nuevas estrategias tratando de lograr la mayor eficacia y eficiencia posible, propiciando su sostenibilidad.

\section{REFERENCIAS BIBLIOGRÁFICAS}

1. DIRESA-ICA, Oficina de Epidemiologia. Sala de situación Dengue. Ica-Perú; 05 de julio de 2015.

2. Deparis X, Murgue B, Roche C, Cassar O, Chungue E. Changing clinical and biological manifestations of dengue during ten dengue-2 epidemic in French Polynesia in 1996/ 1997 - description and analysis in prospective study. Trop Med Int Health. 1998;3(11):859-65.

3. Morrison AC, Minnick SL, Rocha C, Forshey BM, Stoddard ST, Getis A, et al. Epidemiology of dengue virus in Iquitos, Peru 1999 to 2005: interepidemic and epidemic patterns of transmission. PLoS Negl Trop Dis. 2010;4(5):e670. doi: 10.1371/journal.pntd.0000670.

4. Organización Mundial de la Salud, Programa Especial para Investigación y Capacitación en Enfermedades Tropicales. Dengue: Guía para el diagnóstico, tratamiento, prevención y control. Bolivia: OMS; 2009.

5. Vilcarromero S, Casanova W, Ampuero JS, Ramal-Asayag C, Siles C, Díaz G, et al. Lecciones aprendidas en el control de Aedes aegypti para afrontar el dengue y la emergencia de chikungunya en Iquitos, Perú. Rev Peru Med Exp Salud Pública. 2015;32(1):172-8.

Correspondencia: Tania Acevedo Villar

Dirección: Calle Los Pacaes B-14 D. Ica, Perú

Teléfono: (051) 942433952

Correoelectrónico: tania.acevedo5@gmail.com

\section{¿QUÉ SABEMOS SOBRE LA FALTA DE RESPETO Y MALTRATO DURANTE LA ATENCIÓN DEL PARTO EN EL PERÚ?}

\author{
WHAT DO WE KNOW ABOUT THE LACK OF \\ RESPECT AND ABUSE DURING CHILDBIRTH \\ CARE IN PERU?
}

\author{
Reneé Montesinos-Segura, ${ }^{1, a}$, \\ Álvaro Taype-Rondán ${ }^{2, b}$
}

Sr. Editor. El maltrato que sufre la mujer durante el parto es reconocido como un problema de gran importancia ${ }^{(1)}$. El término que se suele usar para estos casos es "Falta de respeto y maltrato durante la atención del parto". Aunque recientemente se ha introducido como sinónimo el término "violencia obstétrica" en el ámbito legal de Venezuela ${ }^{(2)}$. Estas definiciones son amplias, y van más allá de la violencia física, como se detalla en la Tabla 1.

Estas malas prácticas afectan física y psicológicamente a la mujer, quien llega a temer la atención obstétrica que se brinda en los establecimientos de salud. Esto disminuye las probabilidades de buscar un parto institucionalizado en su siguiente embarazo, aumentando así el riesgo de mortalidad materna, en especial en países de bajos y medianos ingresos, en los que este temor se suma a otras barreras de acceso como el costo, la distancia y la falta de información.

La prevalencia de la falta de respeto y maltrato durante la atención del parto varía entre países, posiblemente debido a reales diferencias en la atención o a diferencias en los instrumentos y metodologías utilizadas para recolectar la información ${ }^{(3)}$.

En el Perú, la Encuesta Demográfica y de Salud Familiar 2014 señaló que si bien la mortalidad materna se redujo en un $64,9 \%$ entre los años $1990-2010$ al pasar de 265 a 93 muertes maternas por cada 100000 nacidos vivos, estos resultados aún se encuentran distantes de la meta de menos de 66,3 defunciones por cada 100 000 nacidos vivos establecida para el 2015. Además, el $89,2 \%$ de partos fueron atendidos en un establecimiento

\footnotetext{
Asociación Científica de Estudiantes de Medicina Humana del Cusco. Facultad de Medicina, Universidad Nacional de San Antonio Abad del Cusco, Cusco, Perú.

2 Facultad de Medicina Humana, Universidad de San Martín de Porres, Lima, Perú

a Estudiante de Medicina; ${ }^{\mathrm{b}}$ médico cirujano Recibido: 24-05-15 Aprobado: 03-06-15
}

Citar como: Montesinos-Segura R, Taype-Rondán A. ¿Qué sabemos sobre la falta de respeto y maltrato durante la atención del parto en el Perú? [carta]. Rev Peru Med Exp Salud Publica. 2015;32(3):608-10. 